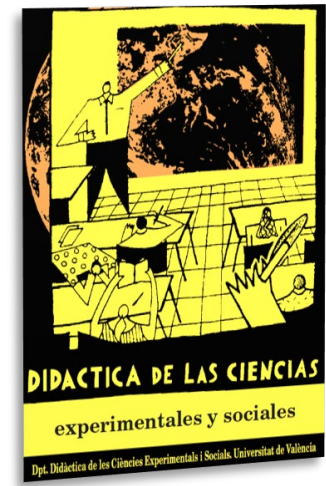

\title{
Las TIC en la didáctica de las ciencias en el ámbito español: revisión sistemática en relación con el tratamiento de competencias digitales
}

\section{ICT in science didactics in Spain: a systematic review in relation to the treatment of digital skills}

DOI: $10.7203 / D C E S .41 .20260$

\author{
Juan José Marrero Galván \\ Universidad de La Laguna, jmarrerg@ull.edu.es \\ ORCID iD: https://orcid.org/0000-0002-7563-0387 \\ Miguel Ángel Negrín Medina \\ Universidad de La Laguna, mnegrinm@ull.edu.es \\ ORCID iD: https://orcid.org/0000-0002-9403-8215 \\ Paula González Pérez \\ gperezpaula@gmail.com \\ ORCID iD: https://orcid.org/0000-0003-0445-9196
}

\begin{abstract}
RESUMEN: Las TIC han generado nuevas posibilidades en la enseñanza de las ciencias, pero su integración efectiva en el aula supone un reto, lo que pone de manifiesto la importancia del desarrollo competencial digital de los docentes. Con el fin de conocer la incorporación de las TIC en la enseñanza de las ciencias en el ámbito educativo español, se ha realizado una revisión bibliográfica en seis revistas de Didáctica de las Ciencias Experimentales de reconocido prestigio. Los resultados muestran que en el periodo de marzo de 1983 a diciembre de 2019 se han publicado 202 artículos TIC. El análisis y categorización de estas publicaciones permite concluir aportes significativos en áreas como la pedagogía digital, el compromiso profesional y los recursos digitales; aunque también se evidencia que se requiere mayor atención en relación con la evaluación y promoción de la competencia digital de los estudiantes.
\end{abstract}

Palabras Clave: TIC, estudio bibliométrico, didáctica de las ciencias, investigación didáctica, competencia digital docente

ABSTRACT: ICTs have created new possibilities in science teaching, but their effective integration in the classroom is a challenge, which highlights the importance of teachers' digital competences development. In order to know about the incorporation of ICTs in science teaching in Spanish education, a bibliographic review has been carried out in six prestigious journals of Science Education. The results show that in the period from March 1983 to December 2019, 202 articles referring to ICTs have been published. The analysis and categorization of these publications allow us to conclude that significant contributions have been made in areas such as digital pedagogy, professional commitment, and digital resources; although it is also relevant that greater attention is required in relation to the evaluation and promotion of students' digital competence.

KEYWORDS: ICT, bibliometric study, science education, didactic research, teacher digital competence

Fecha de recepción: febrero de 2021

Fecha de aceptación: mayo de 2021 


\section{INTRODUCCIÓN}

Las tecnologías de la información y la comunicación (TIC) son más que una simple moda en la sociedad actual y se admite, de forma más o menos generalizada, su creciente repercusión en todos los ámbitos, especialmente en el educativo. Así por ejemplo, según el Instituto Nacional de Tecnologías Educativas y de Formación del Profesorado (INTEF) en el año 2016 entre todos los centros escolares españoles se habría pasado de 13 alumnos, de media, por ordenador destinado a tareas de enseñanza y aprendizaje en el curso 2002-2003, a 3 alumnos en el curso escolar 2014-2015; y además, que el 99,9\% de los centros escolares españoles ya disponía de conexión a Internet en ese último curso.

Esta incorporación de las TIC a la enseñanza constituye un nuevo medio de transmisión de conocimientos, así en las últimas décadas, en la escuela se ha considerado esta incorporación desde tres puntos de vista: la formación de los estudiantes como usuarios de estas tecnologías, su incorporación al aula como recurso didáctico que ayuda al profesor y como instrumento de enseñanza (Brincones y Blázquez, 2008).

Son pocas las investigaciones que han analizado la efectividad del uso de ordenadores en la promoción del conocimiento y en la generación de nuevos tipos de aprendizaje (Brandsford, Brown \& Cocking, 1999; Brandsford, Brophy \& Williams, 2000) aunque en los últimos años ya se han publicado algunos resultados sobre evaluación de la enseñanza apoyada con nuevas tecnologías (Hennessy et al., 2007; OCDE, 2010); por el contrario, sí se han publicado en nuestro país estudios dirigidos a la integración de las TIC, así por ejemplo: Pontes (1999), Sanmartí e Izquierdo (2001), Fuentes, Ortega y Delgado (2005), Oñorbe (2014) y Romero y Quesada (2014).

Generalmente las investigaciones o innovaciones se suelen publicar en revistas científicas, razón por la cual, la temática "incorporación de las TIC a la enseñanza” y en concreto a la enseñanza de las ciencias, ha generado una nueva línea editorial o tipología de trabajo.

\section{FUNDAMENTACIÓN}

Este trabajo se fundamenta en el análisis de las interacciones que se presentan entre la Didáctica de las Ciencias Experimentales (DCE) como campo de estudio, las Revistas de DCE como medio de comunicación y las TIC como línea de investigación.

El tratamiento de las dimensiones que surgen de la interacción de estos tres ámbitos permite contextualizar este estudio, intentando presentar un balance entre antecedentes y nuevas aportaciones, que permita conocer en su conjunto el Uso educativo de las Tecnologías de la Información y de la Comunicación (UETIC) en el ámbito de la enseñanza de las ciencias experimentales.

En la figura 1 podemos observar una representación de las interacciones que se producen entre estos ámbitos.

FIGURA 1. Interacciones entre DCE, Revistas y TIC

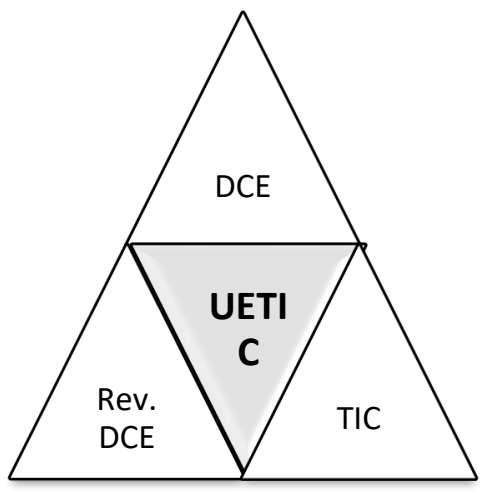

Fuente: elaboración propia 


\subsection{DCE y Revistas}

En la actualidad las revistas científicas del campo educativo juegan un importante papel para hacer llegar información de calidad al profesorado e investigadores. Estas publicaciones promueven por un lado la investigación y la mejora educativa y por otro contribuyen al progreso del conocimiento en el ámbito.

Una buena forma de hacerse una idea general del estado de la investigación en DCE, en relación con un tema, es recurrir a alguna de las revisiones que aparecen en las publicaciones de bibliografía (Prieto y Blanco, 1997). Del mismo modo Castilho (2014, p.1) señala que "las revistas científicas son relevantes medios de difusión y deben ser periódicamente analizadas, porque sus datos cualicuantitativos de producción y de contenido, en conjunto con los artículos que se publican, son potenciales subsidios para las diferentes áreas del conocimiento".

En la literatura científica numerosas son las publicaciones que abordan los estudios de revisión. Por ejemplo, el estudio de Gilbert (1995) ofrece un panorama básico de las publicaciones aparecidas en Studies in Science Education. Al describir el panorama de la revista, desde su inicio, va configurando diferentes campos y ofrece una imagen global del estado de la cuestión y de los aspectos en los que se ha dedicado mayor atención.

Otros autores presentan estudios similares en diferentes revistas de enseñanza de las ciencias, como, por ejemplo: Enseñanza de las Ciencias (Moreira, 1994; López, Salvador y De La Guardia, 1998); Alambique (Del Carmen, 1997); Revista Eureka (Castilho, 2014) e Investigación en la Escuela (García-Gómez, 2016).

Por otra parte, también se han publicado estudios de revisión bibliográfica desde una perspectiva temática, así por ejemplo, Franco, Velasco y Riveros (2017) analizan los trabajos prácticos de laboratorio en la enseñanza de las ciencias; Manchón-Gordón, García-Carmona (2018) la investigación didáctica en el aula de física; Aguilera (2018) las salidas de campo como recurso didáctico para la enseñanza de las ciencias; Ramírez-Montoya y García-Peñalvo (2018) la cocreación e innovación; Toma (2020) instrumentos sobre actitudes hacia la ciencia y, finalmente, SolaMartínez, Caceres-Reche, Romero-Rodríguez y Ramos-Navas-Parejo (2020) la formación del profesorado en TIC que se relacionan con la calidad educativa.

\subsection{Revistas de DCE y las TIC}

Tal como ya se indicó anteriormente, la incorporación de las TIC a la enseñanza y, en concreto, a la enseñanza de las ciencias, ha generado una línea editorial o tipología de trabajo que se refleja no sólo en la gran cantidad de artículos dedicados al tema, sino también por la edición de monográficos por parte de revistas que abordan la integración de las TIC en la escuela.

La finalidad de las monografías es dar cuenta sobre el estado de conocimiento de la temática a la que se dedica, es decir presentar estudios, investigaciones, innovaciones, experiencias, etc, con el objetivo de situar el tema y sus perspectivas de futuro. Por tanto, que las revistas especializadas presenten monográficos sobre las TIC permite reafirmar la importancia de esta línea de investigación.

Algunos ejemplos de monográficos dedicados a las TIC en revistas son:

- Alambique, dedicó el número 19 de 1999 a las "Nuevas tecnologías de la información y la comunicación", el número 50 de 2006 a "Internet en la enseñanza de la ciencia" y el número 76 de 2014 a "Las TIC en el aula".

- Tarbiya, dedicó el número 39 de 2008 a "Las TIC en la enseñanza".

- Investigación en la escuela, dedicó el número 41 de 2000 a "Cultura audiovisual, cultura escolar" y el número 64 de 2008 a "Internet y la investigación escolar" 


\subsection{DCE y las TIC}

La incorporación de las TIC a la escuela ha sido un proceso lento y no siempre se ha realizado de forma idónea, ya que la escasez de recursos, políticas erróneas, inadecuada o escasa formación del profesorado, etc. han dificultado su integración.

Hay que tener presente que el uso de las TIC en el aula por parte del profesorado en ocasiones se ha tenido más presente el aspecto tecnológico que el modelo pedagógico o didáctico en el que se utiliza dicha tecnología (Oñorbe 2014), aunque también la incorporación de las TIC a la enseñanza ha permitido generar nuevas oportunidades para facilitar la enseñanza y el aprendizaje, además de presentar claras ventajas pedagógicas si se utilizan de una forma adecuada (Lelouch, 1998; Marrero y Expósito, 2006; Domínguez, Marrero y Negrín, 2017).

En cuanto al uso de las TIC y su influencia en la enseñanza de las ciencias coincidimos con Pontes (2005a, p.1) cuando señala que:

Las TIC ejercen actualmente una influencia cada vez mayor en la educación científica, tanto en la enseñanza secundaria como en la universitaria, no sólo en lo que respecta a la mejora del aprendizaje de la ciencia por parte de los alumnos de tales niveles, sino que también desempeñan un papel creciente en la formación inicial y permanente del profesorado.

Este mismo autor, analiza las funciones que pueden desempeñar las TIC en la educación científica y los diferentes tipos de recursos informáticos que pueden utilizar el profesorado, mostrando las diferencias entre los recursos informáticos de propósito general y los programas específicos de enseñanza de las ciencias.

Otros autores señalan posibilidades y limitaciones que las TIC pueden llegar a ejercer en la enseñanza de las ciencias; así Giordan (2011) sugiere dos ejes de análisis: uno relacionado con las formas de organización del trabajo y el otro con las formas de representación.

Parece evidente que se necesitaría profundizar en las relaciones que surgen entre los modelos didácticos tradicionales de las ciencias experimentales y los nuevos espacios de enseñanza aprendizaje basados en las TIC, con el objeto de estimular en el profesorado de ciencias experimentales la necesaria reflexión que conlleva su práctica docente en estos nuevos entornos (Marrero y Fernández, 2011), sin olvidar la influencia de la imagen que el alumnado tiene de las TIC en la enseñanza (Solbes et al., 2004)

\subsection{Uso educativo de las TIC (UETIC)}

Los usos educativos de las TIC pueden ser variados y diversos atendiendo a propuestas y criterios de diferentes. Por ejemplo, Coll, Mauri y Onrubia (2008) reflexionan sobre la tipología de los usos educativos de las TIC y realizan una propuesta fundamentada en que pueden funcionar como herramientas psicológicas susceptibles de mediar los procesos inter e intra-psicológicos implicados en la enseñanza aprendizaje, además proponen que las TIC medien entre alumnos, profesores y contenidos, contribuyendo a conformar un contexto de actividad en el que se tienen lugar estas relaciones. A su vez, Solbes, Souto y Traver (2004) analizan el impacto de las TIC en el sistema escolar.

En cuanto a la enseñanza de las ciencias, diversos autores enfatizan la necesidad de analizar las distintas formas de utilizar las TIC y formular propuestas metodológicas orientadas a favorecer el aprendizaje (Pontes, 2005b; Marrero y Expósito, 2006). En este sentido, Romero y Quesada (2014) identifican previamente cuáles son los principales obstáculos que se suelen dar en un aprendizaje significativo en la enseñanza de las ciencias y reflexionan sobre su origen, con el objetivo de valorar hasta qué punto las TIC pueden ayudar a afrontar dichos obstáculos y facilitar así el aprendizaje.

Por otra parte, el confinamiento decretado en España en marzo de 2020, a consecuencia de la pandemia de COVID-19 producida por el virus SARS-CoV-2, puso de manifiesto la desigualdad 
digital existente entre el profesorado, pues un número importante de docentes no propició de manera rápida y eficaz la incorporación de las TIC al proceso de enseñanza y aprendizaje (Martínez-Pérez y Lezcano-Barbero, 2020). Sin embargo, esta desigualdad digital también puso en evidencia la predisposición de ciertos docentes con una competencia digital alta por compartir con aquellos cuya competencia es más débil en sus destrezas y conocimientos (Boix, 2020), produciendo un incremento considerable en la percepción docente de la importancia de las TIC durante y después del confinamiento frente a la que se tenía sobre estas previamente (García, 2020).

En definitiva, la situación producida por la COVID-19, ha puesto de manifiesto la importancia de reflexionar sobre la formación en competencias digitales de profesores y estudiantes así como de la necesaria transformación de los roles docentes (Cabero-Almenara, 2020).

En este contexto de necesaria reflexión surgen algunas preguntas clave que se plantean en este trabajo: 1. ¿En qué medida está presente la temática TIC en las revistas españolas de enseñanza de las ciencias? 2. ¿Con qué competencia digital se relacionan los trabajos publicados? 3. ¿Existen ámbitos competenciales con escases de artículos de referencia? Estas preguntas conllevan a que este estudio de revisión bibliográfica se plantee los siguientes objetivos:

- Primero: determinar los artículos TIC publicados en revistas españolas de DCE, así como indicadores bibliométricos para caracterizar la muestra.

- Segundo: analizar los artículos publicados y distribuirlos en relación con el tratamiento de competencias digitales, de tal forma que permita, por un lado, la consulta de artículos de referencia, y por otro, conocer las competencias que presentan un déficit de publicaciones.

\section{Metodología}

Para abordar los objetivos del estudio se utilizó una revisión bibliográfica sistemática como estrategia para identificar los artículos de DCE relacionados con la temática TIC y que se publican en España.

El proceso de esta revisión está sustentado en las directrices establecidas por la declaración PRISMA para este tipo de revisiones (Liberati et al., 2009; Urrutia y Bonfill, 2010) y en trabajos similares como los de Aguilera (2018); Manchón-Gordón y García-Carmona (2018) y Toma (2020).

\subsection{Criterios de elegibilidad, inclusión, exclusión y fuentes de información}

Se seleccionaron aquellos trabajos, formatos artículos, relacionados con las TIC en el campo de la enseñanza de las ciencias, acotados al ámbito español y publicado por revistas específicas de DCE desde su comienzo editorial. Se excluyeron actas de congresos, capítulos de libros, reseñas u otros.

La selección de revistas se hizo atendiendo a su difusión e indicios de calidad y, por tanto, incluidas en catálogos, directorios y bases de datos de reconocido prestigio (Scopus, JCR-WOS, Scimago, Dialnet). Además, con el objeto de limitar el estudio se decidió también considerar los siguientes criterios: publicaciones de acceso en abierto y disponible en internet para facilitar su consulta, con revisión por pares para asegurar una valoración rigurosa y crítica, una antigüedad de al menos cinco años con el objeto de que se haya definido la línea editorial y su consolidación en el ámbito educativo $\mathrm{y}$, por último, en colaboración con alguna universidad española que garantice su excelencia.

Atendiendo a los criterios señalados, las revistas seleccionadas para su revisión fueron: "Didáctica de las Ciencias Experimentales y Sociales", "Enseñanza de las Ciencias. Revista de Investigación y Experiencias Didácticas", "Investigación en la Escuela", "REEC. Revista Electrónica de Enseñanza de las Ciencias", "Revista Eureka sobre enseñanza y divulgación de las Ciencias" y "Didácticas Específicas". 
El periodo analizado se corresponde desde 1983, año de publicación del primer número de la revista más antigua, hasta diciembre de 2019. Es importante señalar que en el año 2001 sólo eran tres las revistas activas, incorporándose el resto en el periodo 2002-2009.

\subsection{Búsqueda bibliográfica}

Para organizar y orientar las búsquedas se buscó el acrónimo "TIC" o la palabra "digital”, en los títulos de los artículos, el resumen y palabras clave de los trabajos. Dado que no todas las revistas estaban indexadas en las mismas bases de datos, se decidió realizar la búsqueda a través de los propios buscadores de las revistas, pero se detectó que mediante dicho procedimiento no aparecían numerosos trabajos que ya se tenía constancia de su publicación, por lo que se revisó uno a uno todos los números de dichas publicaciones a través de los archivos de las propias revistas, contabilizando todos los artículos publicados y seleccionando aquellos que cumplían los requisitos indicados anteriormente. Este procedimiento resultó ser un proceso lento, laborioso y complejo, pero permitió la minuciosidad necesaria para este tipo de estudio, ya que se detectaron trabajos que no estaban indexados en los buscadores de las revistas pero que tras este análisis pormenorizado se consideró que cumplían los criterios para ser incluidos en la investigación. También hay que reseñar que los buscadores de las revistas permitieron encontrar algunos artículos que habían pasado desapercibidos en el análisis pormenorizado. Finalmente, la comparación entre ambos métodos permitió obtener la muestra final del estudio.

\subsection{Tratamiento de datos}

Una vez seleccionados los trabajos objeto de estudio, se realizó en una primera fase, un análisis cuantitativo de los principales parámetros bibliométricos, utilizando para ello la herramienta ofimática Microsoft Excel 2016.

FIGURA 2. Competencias y descriptores
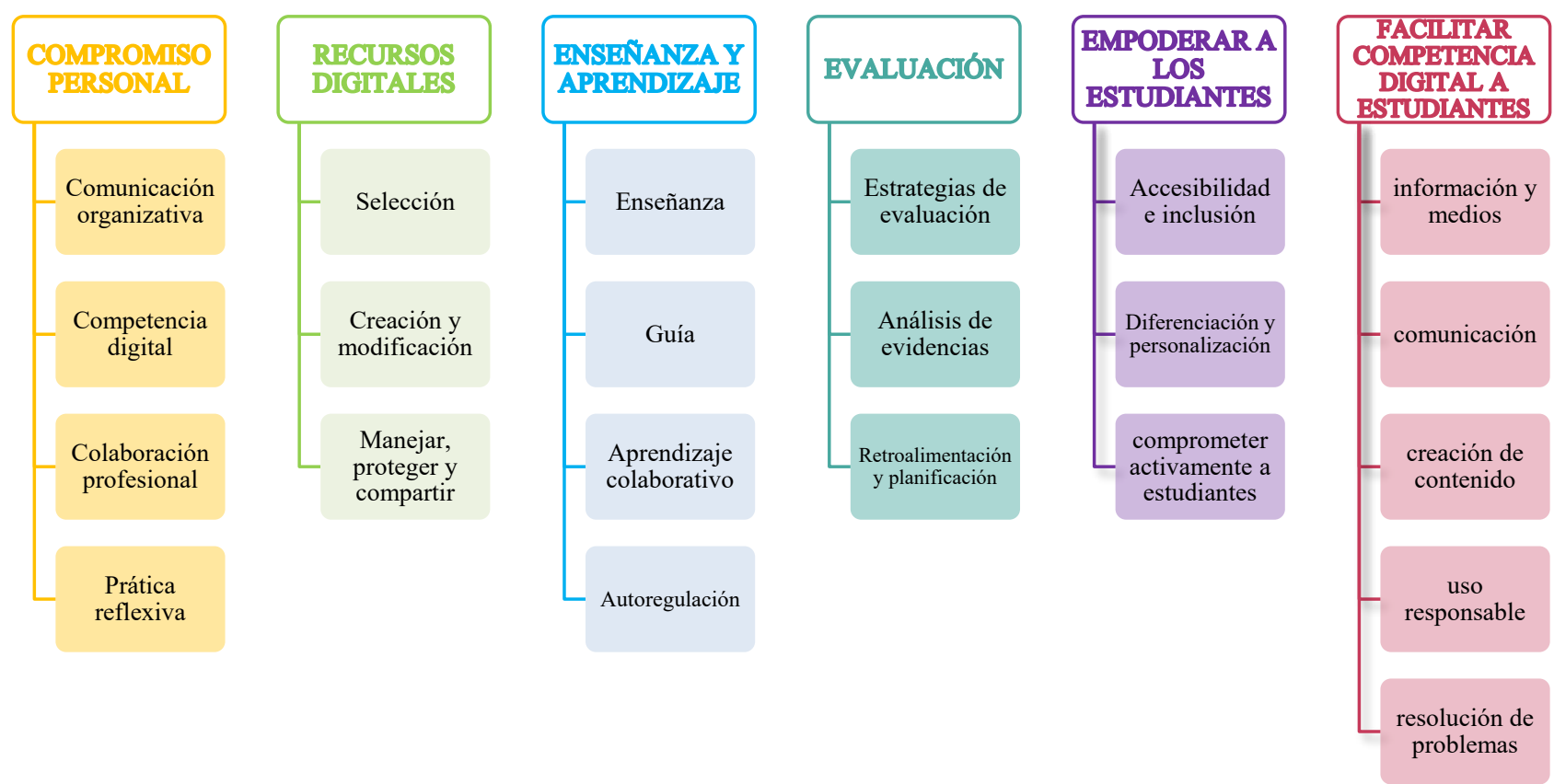

Fuente: adaptación de Redecker \& Punie, 2017 
Los indicadores considerados fueron: frecuencia de artículos por revista y autoría (número de autores/as, sexo y etapa educativa). En una segunda fase se categorizaron los artículos publicados, mediante un análisis cualitativo del contenido en relación con el tratamiento de competencias digitales docentes, para ello, se tuvo en cuenta el título y resumen, y en caso de duda se amplió la lectura al resto del artículo, de tal forma que permitiera a los investigadores determinar su clasificación. Dado que existen diversos marcos, herramientas y programas de formación en relación a las TIC, se decidió tomar como referencia el European Framework for the Digital Competence of Educators (Redecker \& Punie, 2017) marco auspiciado por la Comisión Europea. Dicho marco está constituido por seis áreas: compromiso profesional, recursos digitales, pedagogía digital, evaluación y retroalimentación, empoderar a los estudiantes y facilitar la competencia digital de los estudiantes, que engloban en su conjunto a veintidós ámbitos de competencias digitales para los docentes, lo que permite clasificar la muestra. A modo de ejemplo, el área recursos digitales, se subdivide en los siguientes descriptores: selección de recursos digitales, creación y modificación de recursos digitales y administrar, proteger y compartir recursos digitales. La figura 2 representa el marco de referencia con las seis áreas competenciales y descriptores de cada una de ellas.

\section{RESUltadoS Y ANÁLISIS}

\subsection{Identificación y selección de estudios}

La búsqueda inicial de artículos a través de las herramientas web de las revistas analizadas se tradujo en 164 artículos, 68 de los cuales atendían a los criterios de selección. La búsqueda pormenorizada en cada de una de las revistas permitió obtener 196 artículos sobre TIC de un total de 3671 publicados. La comparación de los resultados, tras la eliminación de las citas duplicadas, supuso obtener 202 artículos que cumplían los criterios de selección, un 5,5\% del total, que representan la muestra de este estudio.

\subsection{Primera fase: indicadores bibliométricos}

La distribución de artículos publicados por revista se muestra en la tabla 1. Como es lógico los resultados no presentan una distribución homogénea, ya que hay que tener en cuenta, por un lado, la antigüedad de cada revista, la variedad de números anuales publicados y el número de artículos incluidos en estos; por otro, algunas revistas también presentan en sus publicaciones artículos no relacionados directamente con la didáctica de las ciencias experimentales. Las revistas con mayor número de publicaciones son REEDC (34,7\%), REEC (21,3\%) y EC (20,3\%).

TABLA 1. Distribución de Artículos TIC por revista

\begin{tabular}{lcc}
\hline \multicolumn{1}{c}{ Revistas } & Frecuencia & Porcentaje \\
\hline Enseñanza de las Ciencias (EC) & 41 & 20,3 \\
\hline Didáctica de las Ciencias Experimentales y Sociales (DCES) & 16 & 7,9 \\
\hline Didácticas Específicas (DE) & 5 & 2,5 \\
\hline Investigación en la Escuela (IE) & 27 & 13,4 \\
\hline Revista Electrónica de Enseñanza de las Ciencias (REEC) & 43 & 21,3 \\
\hline Revista Eureka sobre enseñanza y divulgación de las Ciencias (REEDC) & 70 & 34,7 \\
\hline Total & 202 & 100 \\
\hline
\end{tabular}

Fuente: elaboración propia 
La distribución de los artículos publicados por año y por cada una de las revistas se muestra en la figura 1. Se puede observar que las revistas REEDC y REEC mantienen de forma más o menos continua la publicación de artículos con esta temática, por lo que se podría inferir que en su línea editorial está relativamente consolidada. En el resto de las revistas, es decir EC, DCES, IE y DE se aprecia algunos años en los que no han publicado artículos sobre TIC, reflejando una variabilidad mayor en la frecuencia de publicación. Hay que destacar que ya en la década de los 80, las revistas EC y DCES publicaron artículos con esta temática. En la siguiente década la revista EC publicó nueve artículos, la revista DCES cinco e IE uno. A partir del año 2000, es cuando ha habido el mayor número de publicaciones y ha ido creciendo de forma continua.

FIGURA 3. Artículos con temática TIC

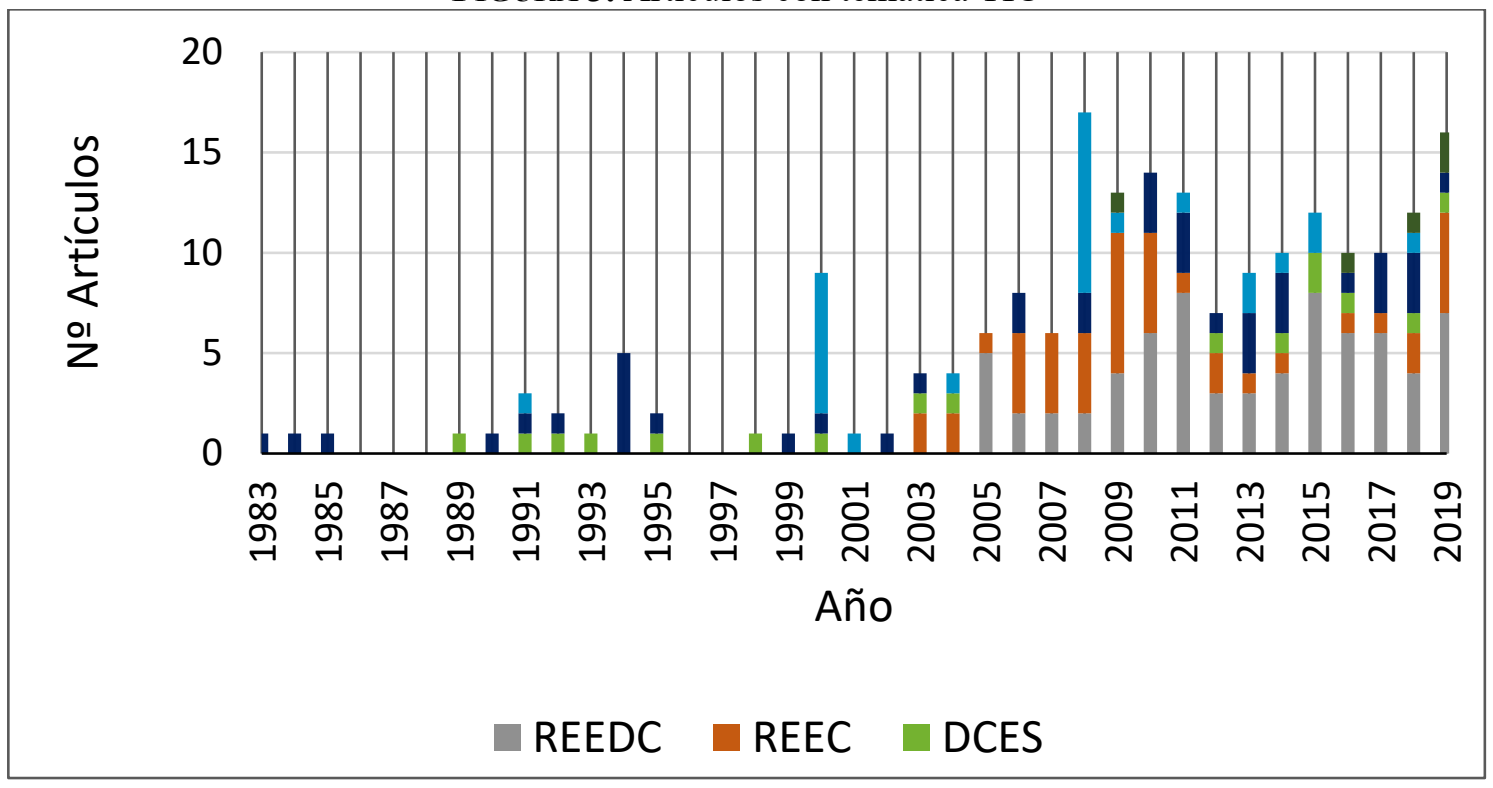

Fuente: elaboración propia

En cuanto a la autoría de los artículos, han participado en su elaboración 468 investigadores, de los cuales, 287 son hombres (61,3\%) y 181 mujeres (38,7\%). En la tabla 2, se muestra la distribución de artículos por número de autores y sexo.

TABLA 2. Distribución de artículos por número de autores y sexo

\begin{tabular}{ccccccc}
\hline $\mathrm{N}^{\circ}$ Autores & Frecuencia & $\%$ & Hombres & \% Hombres & Mujeres & \% Mujeres \\
\hline 1 & 59 & 29,2 & 48 & 10,3 & 11 & 2,4 \\
\hline 2 & 61 & 30,2 & 70 & 15,0 & 52 & 11,1 \\
\hline 3 & 53 & 26,2 & 92 & 19,7 & 67 & 14,3 \\
\hline 4 & 19 & 9,4 & 45 & 9,6 & 31 & 6,6 \\
\hline 5 & 9 & 4,5 & 29 & 6,2 & 16 & 3,4 \\
\hline 7 & 1 & 0,5 & 3 & 0,6 & 4 & 0,9 \\
\hline Total & 202 & 100 & 287 & 61,3 & 181 & 38,7 \\
\hline
\end{tabular}

Fuente: elaboración propia

La mayoría de los artículos están escritos por pocos autores (entre uno y tres). Así, por ejemplo, se detectaron 61 artículos firmados por dos autores (30,2\%), 70 hombres $(15,0 \%)$ y 52 mujeres $(11,1 \%)$, mientras que 59 artículos tuvieron autoría única, siendo firmados únicamente por 11 mujeres 
$(2,4 \%)$ frente a 48 hombres (10,3\%). Finalmente, por tres autores se detectaron 53 publicaciones, en las que participan $92(19,7 \%)$ hombres y 67 (14,3\%) mujeres. La autoría del resto de artículos también fue copada por hombres lo que se evidencia, a nivel general, la existencia de una brecha de género significativa.

En la tabla 3 se puede apreciar la frecuencia de artículos por etapa educativa. La mayor parte de los trabajos están dirigidos a la educación secundaria, 72 (35,6\%), o a la universidad, 60 (29,7\%), si bien 62 de ellos $(30,7 \%)$ que se pueden considerar para dos o más etapas educativas y que se han agrupado en una categoría genérica. Es reseñable el escaso número de artículos dirigidos a la etapa infantil-primaria, con tan sólo 8 (4,0\%), que evidencia un déficit de investigaciones para este ámbito.

TABLA 3. Distribución de artículos por etapa educativa

\begin{tabular}{lcc}
\hline \multicolumn{1}{c}{ Etapa } & Frecuencia & Porcentaje \\
\hline Universidad & 60 & 29,7 \\
\hline Secundaria & 72 & 35,6 \\
\hline Infantil y primaria & 8 & 4,0 \\
\hline Genérico & 62 & 30,7 \\
\hline Total & 202 & 100 \\
\hline \multicolumn{4}{c}{ Fuente: elaboración propia }
\end{tabular}

\subsection{Segunda fase: categorización}

En la tabla 4 se distribuyen los artículos de la muestra en función de las áreas contempladas en el marco European Framework for the Digital Competence of Educators (DigComEdu). Aunque hay que tener en cuenta que algunos de ellos, dada su temática y desarrollo, podrían tener cabida en dos o más áreas, por lo que se consideró su distribución en función del área predominante.

TABLA 4. Distribución de artículos por áreas

\begin{tabular}{lcc}
\hline \multicolumn{1}{c}{ Áreas DigComEdu } & Frecuencia & Porcentaje \\
\hline a) Compromiso profesional & 47 & 23,3 \\
\hline b) Recursos digitales & 26 & 12,9 \\
\hline c) Pedagogía digital & 114 & 56,4 \\
\hline d) Evaluación y retroalimentación & 1 & 0,5 \\
\hline e) Empoderar a los estudiantes & 10 & 5,0 \\
\hline f) Facilitar la competencia digital de los estudiantes & 4 & 2,0 \\
\hline Total & 202 & 100 \\
\hline
\end{tabular}

Fuente: elaboración propia

La mayor parte de los artículos se corresponden con tres áreas: 47 sobre compromiso profesional $(23,3 \%), 26$ en recursos digitales $(12,9 \%)$ y 114 sobre pedagogía digital $(56,4 \%)$. En el resto de las áreas se obtuvieron 15 trabajos que representan el 7,5\% del total.

Desde un punto de vista del análisis global de competencias en función de los intervinientes en los procesos educativos, la mayoría de los artículos 151 (74,7\%) abordan las competencias pedagógicas de los educadores (resultante de la suma de las áreas b, c, d y e), 47 (23,3\%) respecto a las competencias profesionales de los educadores, y finalmente tan solo $4(2 \%)$ en relación con las competencias de los estudiantes. Estos datos son significativos, ya que muestra claramente las líneas 
prioritarias de publicación, pero también la necesidad de que las revistas científicas e investigadores no solo aborden la enseñanza de las ciencias sino que también se involucren más en estimular las competencias digitales de los estudiantes, pues difícilmente se puede desligar la una de la otra, más aún si tenemos en cuenta la perspectiva de un aprendizaje integrado.

A continuación, en el análisis por área y distribución de los artículos en base a los descriptores de cada una ellas, se citan algunos artículos, con el objeto, por un lado, de entender la clasificación realizada, y por otro, disponer de trabajos de consulta en dichos ámbitos. En aquellas áreas donde el número de publicaciones es excesivo, se indicarán algunas de las referencias más recientes, atendiendo al criterio de que el tiempo es un aspecto determinante cuando se habla de tecnología.

\section{Área compromiso profesional}

De los 47 artículos asociados a esta área, 5 hacen referencia a la colaboración profesional. En concreto, a la utilización de tecnologías digitales para colaborar con otros educadores, compartiendo conocimientos y experiencias (Tobin, 1999; Marín, 2010); innovando prácticas pedagógicas de manera colaborativa (Herzel y Cañal, 2008; López, 2008) o utilizando redes colaborativas profesionales como fuente de desarrollo profesional (Negre y Marín, 2014).

Otros 5 artículos tratan el desarrollo profesional continuo digital, desde el punto de vista del uso de fuentes y recursos digitales para el desarrollo profesional continuo (Gras-Martí, Cano-Villalba, y Cano, 2004; García, Greca, y Meneses, 2008; Pereira, Ostermann, y Cavalcanti, 2009; Santos y Miranda, 2009) y Pagani y Da Silva, 2009).

Otros 37 artículos se relacionan con la práctica reflexiva pues, en mayor o menor medida, reflexionan, evalúan críticamente y desarrollan activamente la propia práctica pedagógica digital o de la comunidad educativa. Algunos de estos estudios ponen de manifiesto el pobre conocimiento de la tecnología, así como de su poder pedagógico por parte de los docentes universitarios (Marcelo, Yot y Perera, 2016), incidiendo en la brecha digital docente (Carrapiço, 2018) y el poco uso de las TIC para el trabajo escolar en las aulas (Gómez, Cañas, Gutiérrez y Martín-Díaz, 2014). También se destaca que la tecnología no garantiza una mejora en la calidad de la educación científica si no va acompañada de una adecuada formación del profesorado y de estrategias de enseñanza aprendizaje que posibiliten la integración de las TIC de forma adecuada (Ruíz et al. 2019; Solbes et al. 2018).

No se aprecian artículos relacionados con la comunicación de la organización, es decir, utilizar las tecnologías digitales para mejorar la comunicación organizacional con estudiantes, familias y terceros.

\section{Área recursos digitales}

De los 26 artículos clasificados en esta área, en 15 se identifican, evalúan y seleccionan recursos digitales para la enseñanza y el aprendizaje. Por ejemplo, Ojeda-Barceló, Gutiérrez-Pérez y PeralesPalacios (2017) realizan un estudio y clasificación de los medios TIC que están a disposición de los educadores ambientales o profesionales de la educación para su tarea docente, mientras que Frozza y Dos Santos (2019) analizan software para la enseñanza de la Química en la educación superior.

Otros 8 artículos hacen referencia a la creación o co-creación de nuevos recursos educativos digitales. En este sentido, Ojeda-Barceló, Gutiérrez-Pérez y Perales-Palacios (2011) presentan un programa virtual, describiendo los aspectos más relevantes para su diseño, desarrollo y puesta en marcha; del mismo modo Giménez, Pagés y Martínez (2011) diseñan y desarrollan un juego educativo para ordenador sobre enfermedades tropicales y salud internacional.

Así mismo, 3 artículos (Fejes, Infante-Malachias, Navas y Nunes, 2008; Domènech et al. 2012; Sandia, Pérez y Rivas, 2019) hacen hincapié en el diseño de recursos y planificación de su uso, considerando aspectos como: el objetivo de aprendizaje específico, el contexto, el enfoque pedagógico o el grupo de estudiantes al que van dirigidos. 
En relación con administrar, proteger y compartir recursos digitales no le es asociado artículo alguno, dado que en términos generales los artículos analizados no van dirigidos a la protección eficaz de los contenidos digitales sensibles, al respeto de las normas de privacidad y derechos de autor o a la comprensión del uso y creación de licencias.

\section{Área pedagogía digital}

A esta área se asocian la mayoría de los trabajos analizados, en concreto 114, lo que representa un $56,4 \%$ del total.

En relación con la enseñanza, esta parece ser el ámbito predominante, ya que se le relacionan 105 artículos, lo que resulta, además, coherente con las políticas o líneas editoriales de estas publicaciones pues, 56 trabajos tratan sobre la integración de dispositivos y recursos digitales en el proceso de enseñanza a fin de mejorar su eficacia en la práctica. En este sentido, Martínez, Ibarra, Pérez y Figueres (2016) presenta el uso del Sistema de Información Geográfica (SIG) de software libre para la enseñanza de la Biología y Geología de $4^{\circ}$ de ESO; Di Laccio et al. (2017) ejemplifican el uso de teléfonos inteligentes (smartphones) para la enseñanza del efecto Doppler; mientras que Martins y Campos (2019) preparan un material didáctico basado en las TIC para la enseñanza de la ecología.

Por otra parte, 49 artículos abordan la experimentación y desarrollo de nuevos formatos y métodos pedagógicos de instrucción. Así, Gómez, Fernández y González (2019) muestran un estudio de la evolución de los modelos mentales sobre fosilización tras el proceso de enseñanza y aprendizaje utilizando determinadas estrategias docentes, mientras que Villalustre, Del Moral y Neira-Piñeiro (2019) identifican las posibilidades didácticas, percibidas por los docentes, de la Realidad Aumentada para el aprendizaje científico en educación primaria y su posible implementación en la enseñanza de ciencias; estos autores detectan serias dificultades como la escasa formación docente y la precariedad de los recursos tecnológicos.

De los 9 artículos del área de pedagogía digital, uno (Ardura y Zamora, 2014) se asocia al descriptor guía, ya que aborda la utilización de herramientas y servicios digitales para mejorar la interacción con los estudiantes, ofrecer orientación o asistencia. Otros 6 artículos del ámbito aprendizaje colaborativo, tratan la utilización de las tecnologías digitales para fomentar y mejorar las estrategias de aprendizaje colaborativo. En este sentido, Duarte y Da Purificação (2017) analizan estrategias mediadas por tecnologías en redes educativas para resolución abierta de preguntas de Ciencias Naturales.

Finalmente, en 2 artículos relacionados con el aprendizaje auto-dirigido (Navas, 2011; Barreto y Barreto, 2013), se abordan la utilización de las tecnologías digitales para apoyar los procesos de aprendizaje auto-dirigidos, es decir, para permitir que los estudiantes planifiquen, supervisen y reflexionen sobre su propio aprendizaje.

\section{Resto de áreas}

Tal como se indicó anteriormente, a las áreas de evaluación y retroalimentación, empoderar a los estudiantes y facilitar su competencia digital, sólo se les asocia 15 trabajos, lo que representa un $7,5 \%$ en su conjunto.

En el área de evaluación y retroalimentación, sólo 1 artículo (Hernández, 2008) se vincula con el ámbito de estrategias de evaluación y ninguno para el ámbito de analizar pruebas y el de retroalimentación y planificación.

A su vez, en el área de empoderar a los estudiantes, los 10 artículos detectados, se pueden asociar a su participación activa, ya que tratan de la utilización de herramientas digitales para fomentar el compromiso activo y el pensamiento creativo de los estudiantes con un tema (Marques y Reis, 2017), el fomento de competencias transversales (Marrero-Galván, 2015; Esquivel, 2019), la 
expresión creativa (Manso y Ezquerra, 2014), o abrir el aprendizaje a contextos del mundo real (Enríquez y Aragón, 2018). Por otro lado, no se asocian trabajos a los ámbitos de accesibilidad e inclusión y al de la diferenciación y personalización. En el área de facilitar la competencia digital de los estudiantes, sólo se vinculan 2 artículos (Fuentes y Monereo, 2008; Valverde-Crespo y GonzálezSánchez, 2016) con el ámbito información y alfabetización mediática, ya que tratan de la incorporación de actividades de aprendizaje que requieren que los estudiantes articulen las necesidades de información, encuentren información y recursos en entornos digitales. Otros 2 trabajos (Domènech, 2015; Manso, Ezquerra, Burgos y Mafokozi, 2019) se asocian con la creación de contenido digital, pues incorporan tareas/deberes y actividades de aprendizaje que requieren que los estudiantes se expresen a través de medios digitales. Finalmente, no se asocian artículos con el resto de los ámbitos del área, es decir con la comunicación y colaboración digital, el bienestar y la solución digital de problemas.

\section{CONCLUSIONES}

Los resultados del estudio sugieren que:

Pregunta 1. Las TIC son importantes en el campo de la Didáctica de las Ciencias Experimentales en el panorama español y se evidencia por el número de artículos $(5,5 \%$ del total) publicados en las revistas analizadas, especialmente en la última década. Las revistas más prolíferas en esta temática son REEDC, REEC y EC, dado que el inicio editorial de cada una ha sido diferente y el número de publicaciones se deben considerar en su conjunto. La mayoría de los artículos están escritos entre uno y tres autores, en su mayoría hombres, reflejando que existe cierta brecha de género. Es importante señalar la escasez de publicaciones dirigidas específicamente a la etapa de infantilprimaria $(4,1 \%)$, ya que la mayoría están dirigidos a secundaria $(35,2 \%)$ o al ámbito universitario $(30,6 \%)$, aunque es cierto que hay un alto porcentaje $(30,1 \%)$ de artículos aplicables a cualquier etapa educativa.

Pregunta 2. En cuanto a la tipología de los artículos, la mayoría $(56,4 \%)$ hace referencia a la pedagogía digital y, en concreto, al ámbito de la enseñanza, evidenciando que es el área a la que más atención se le ha prestado por parte de los investigadores. También es importante el número de artículos relacionados con el compromiso profesional (23,3\%), de los que hay que señalar 37 artículos dirigidos a la práctica reflexiva, ya que éstos permiten analizar aspectos clave que pueden desempeñar las TIC en la educación científica y conocer los factores que influyen en la conversión de estas tecnologías en herramientas para el aprendizaje (Pontes, 2005b; Sancho, 2008).

Pregunta 3. El estudio también muestra el déficit de artículos relacionados con las áreas de evaluación, empoderar a los estudiantes y facilitar la competencia digital de los estudiantes, mostrando la necesidad de prestar mayor atención a estas competencias.

\section{LIMITACIONES}

Obviamente, el estudio presenta algunas limitaciones a tener en cuenta. Primero, se analizan solo los artículos de seis revistas, no considerando otras publicaciones y tipologías de trabajos. Estas revistas difieren en cuanto inicio editorial, número de publicaciones y también algunas incluyen artículos de otros campos de estudio. Segundo, dado el período de tiempo que abarca el estudio, el número de artículos obtenidos ha sido excesivo, pero se ha considerado necesario para determinar con perspectiva el estado de la cuestión. Tercero, los datos analizados se han obtenido a través de las páginas webs de las revistas (última consulta realizada abril de 2020), por lo que puede haber variaciones si existen actualizaciones o nuevas publicaciones. Cuarto, la distribución de los artículos según DigComEdu tiene cierto grado de subjetividad, ya que muchos tratan diferentes aspectos y su categorización puede generar debate, pues sus descriptores parecen abrir la puerta para ello. 
Por lo que, teniendo en cuenta todas estas limitaciones las conclusiones del estudio se deben considerar con cierta relatividad. Aun así, la información que aporta puede ser relevante para investigadores y profesionales de la educación, ya que analiza una muestra de publicaciones orientadas a las distintas competencias digitales docentes, algunas de las cuales requieren mayor investigación.

\section{Referencias}

Aguilera D. (2018). La salida de campo como recurso didáctico para enseñar ciencias. Una revisión sistemática. Revista Eureka sobre Enseñanza y Divulgación de las Ciencias, 15(3), 3103. DOI: 10.25267/Rev Eureka ensen divulg cienc.2018.v15.i3.3103

Ardura, D. y Zamora, Á. (2014). ¿Son útiles los entornōs virtuales de aprendizaje? Evaluación de una experiencia en la enseñanza y el aprendizaje de la Relatividad. Revista Eureka sobre Enseñanza y Divulgación de las Ciencias, 11(1), 83-93. DOI: 10.25267/Rev_Eureka_ensen_divulg_cienc.2014.v11.i1.08

Barreto, A. y Barreto, M. (2013). Implantación de las TIC en la materia Química Inorgánica. Enseñanza de las Ciencias, 31(2), 253-268. DOI: 10.5565/rev/ec/v31n2.702

Boix, S. (2020). Competencias de profesores y alumnos españoles de Educación Secundaria Obligatoria durante la pandemia del COVID-19. Boletín Opiniones Iberoamericano en Educación, 2 (12), 29-30. Recuperado de: http://ojs.umc.cl/index.php/bolibero/issue/view/11

Bransford, J., Brophy, S. \& Williams, S. (2000). When Computer Technologies Meet the Learning Sciences: Issues and Opportunities. En A. García y M. Gil (2006). Entornos constructivistas de aprendizaje basados en simulaciones informáticas. Revista Electrónica de Enseñanza de las Ciencias, 5 (2), 304-322.

Bransford, J., Brown, A. \& Cocking, R. (Ed) (1999). How people learn: Brain, mind, experience, and school. En A. García y M. Gil (2006). Entornos constructivistas de aprendizaje basados en simulaciones informáticas. Revista Electrónica de Enseñanza de las Ciencias, 5 (2), 304-322.

Brincones, I. y Blázquez, J. (2008). Diseño de cursos que incorporan la formación con TIC: aspectos metodológicos. Tarbiya, 39, 13-40.

Cabero-Almenara, J. (2020). Aprendiendo del tiempo de la COVID-19. Revista Electrónica Educare, 24 (suplemento especial), 1-3. DOI:10.15359/ree.24-S.2

Carrapiço, F. (2018). Condicionalismos e potencialidades do uso das TIC, no 1o ciclo do ensino básico, no Algarve (Portugal). Uma visão dos professores. Investigación en la Escuela, 95, 6380. DOI: 10.12795/IE.2018.I95.5

Castilho, J. (2014). Un perfil cienciométrico de Revista Eureka sobre Enseñanza y Divulgación de las Ciencias (2004-2013). Revista Eureka sobre Enseñanza y Divulgación de las Ciencias, 12(2), 237-248.

Coll, C., Mauri, T., y Onrubia, J. (2008). La utilización de las tecnologías de la información y la comunicación en la educación: Del diseño tecno-pedagógico a las prácticas de uso. En C. Coll, y C. Monereo (Eds.), Psicología de la educación virtual. Aprender y enseñar con las tecnologías de la información y la comunicación (pp.74-103). Madrid, España: Ediciones Morata.

Del Carmen, L. (1997). Tres años de Alambique. Alambique. Didáctica de las Ciencias Experimentales, 13, 5-8.

Di Laccio, J., Vitale, G., Alonso-Suárez, R., Pérez, N. y Gil, S. (2017). Estudio del Efecto Doppler usando Smartphones. Revista Eureka sobre Enseñanza y Divulgación de las Ciencias, 14(3), 637-646. DOI: 10.25267/Rev_Eureka_ensen_divulg_cienc.2017.v14.i3.09

Domènech, J. (2015). Eppur si muove: una secuencia contextualizada de indagación y comunicación científica sobre el sistema astronómico Sol-Tierra. Revista Eureka sobre Enseñanza y 
Divulgación de las Ciencias, 12(2), 328-340. DOI: 10.25267/Rev_Eureka_ensen_divulg_cienc.2015.v12.i2.07

Domènech, J., Besson, I., Merlo, M., Puigcerver, M. y Solé, M. (2012). Genes, memes y bits: el cómo y el porqué de cinco recursos web sobre genética. Revista Electrónica de Enseñanza de las Ciencias, 11 (1), 43-58.

Domínguez, J.D., Marrero, J.J. y Negrín, M.A. (2017). La sinergia entre la competencia lingüística y la competencia clave en ciencia y tecnología a través del enfoque de aula invertida. El Bucio: revista digital del CEP Tenerife Sur, (22), 53-62.

Duarte, W. y Da Purificação, F. (2017). Trabalho colaborativo no ensino de física mediado por tecnologias educacionais em rede para resolução de problemas. Revista Electrónica de Enseñanza de las Ciencias, 16 (1), 47-68.

Enríquez, J. y Aragón, L. (2018). Creación de una infografía colaborativa para promover el consumo de fruta en la etapa de infantil. Didácticas Específicas, 19, 55. DOI: 10.15366/didacticas2018.19.005

Esquivel, T. (2019). Propuesta educativa para la alfabetización científica sobre salud en bachillerato. Didácticas Específicas, (20), 7. DOI: 10.15366/didacticas2019.20.001

Fejes, M., Infante-Malachias, M., Navas, A. y Nunes, C. (2008). Proceso de autoría/creación de simulaciones realizado por profesores de química: una experiencia de capacitación en servicio. Enseñanza de las ciencias: revista de investigación y experiencias didácticas, 26(2), 281-290.

Franco, R., Velasco, M., y Riveros, C. (2017). Los trabajos prácticos de laboratorio en la enseñanza de las ciencias: tendencias en revistas especializadas (2012-2016). TED: Tecné, Episteme y Didaxis, 41, 37-56. DOI: 10.17227/01203916.6031

Frozza, E. y Dos Santos, B. (2019). Avaliação de software educacionais para o ensino da Química em nível superior. Revista Electrónica de Enseñanza de las Ciencias, 18(1), 251-273.

Fuentes, J., Ortega, J. y Delgado, M. (2005). Tecnofobia como déficit formativo. Investigando la integración curricular de las TIC en centros públicos de ámbito rural y urbano. Educar, 36, 169180.

Fuentes, M. y Monereo, C. (2008). Cómo buscan información en Internet los adolescentes. Investigación en la Escuela, 64, 45-58. DOI: 10.12795/IE.2008.I64.04

García-Gómez, S. (2016). Análisis bibliométrico de la revista Investigación en la Escuela (19872015). Investigación en la Escuela, 88. Recuperado de: www.investigacionenlaescuela.es/artoculos/R88/R88-2.pdf

García, J., Greca, I. y Meneses, J. (2008). Comunidades virtuales de práctica para el desarrollo profesional docente en Enseñanza de las Ciencias. Revista Electrónica de Enseñanza de las Ciencias, 7 (2), 439-462.

García, M. (2020). Covid-19 y formación inicial del profesorado. Revista Trabajadores de la Enseñanza, 376, 22-23. Recuperado de: https://fe.ccoo.es/dbd92e62e5a2027c7a1c5f24ae37c926000063.pdf

Gilbert, J. (1995). Studies and Fields: Directions of Research in Science Education. Studies in Science Education, 25, 179-197.

Giménez, C., Pagés, C., \& Martínez, J. (2011). Diseño y desarrollo de un juego educativo para ordenador sobre enfermedades tropicales y salud internacional: una herramienta docente más de apoyo al profesor universitario. Revista Eureka sobre Enseñanza y Divulgación de las Ciencias, 8 (2), 221-228. DOI: 10.25267/Rev_Eureka_ensen_divulg_cienc.2011.v8.i2.09

Giordan, M. (2011). Diseño de ambientes virtuales de aprendizaje $\overline{\text { de }}$ la química bajo una perspectiva sociocultural. Alambique. Didáctica de las Ciencias Experimentales, 69, 52-66.

Gómez, M., Cañas, A, Gutiérrez, J. y Martín-Díaz, M. (2014) Ordenadores en el aula: ¿estamos preparados los profesores? Enseñanza de las Ciencias, 32(2), 239-250. 
Gómez, R., Fernández y González, F. (2019). Evolución de los modelos mentales sobre fosilización tras el proceso de enseñanza-aprendizaje. Revista Eureka Sobre Enseñanza Y Divulgación De Las Ciencias, 16 (2), 2102.DOI: 10.25267/Rev_Eureka_ensen_divulg_cienc.2019.v16.i2.2102

Gras-Martí, A., Cano-Villalba, M. y Cano, C. (2004). Cursos de TIC per al professorat: anàlisi comparatiu de les modalitats presencial, semipresencial i no presencial. Revista Electrónica de Enseñanza de las Ciencias, 3(1), 48-72.

Hennessy, S., Wishart, J., Whitelock, D., Deaney, R., Brawn, R., Velle, L., Mcfarlane, A., Ruthven, K. \& Winterbottom, M. (2007). Pedagogical approaches for technology-integrated Science teaching. Computers and Education, 48(1), pp. 137-152.

Hernández, F. (2008). El portafolio electrónico y el aprendizaje para la comprensión en secundaria. Investigación en la Escuela, 64, 59-71. DOI: 10.12795/IE.2008.I64.05

Herzel, C. y Cañal, P. (2008). Internet y las redes colaborativas para la investigación escolar en la educación científica. Investigación en la Escuela, 64, 31-44. DOI: 10.12795/IE.2008.I64.03

INTEF (2016). Indicadores del uso de las TIC en España y en Europa. Recuperado de: http://blog.educalab.es/intef/wp-content/uploads/sites/4/2016/11/2016_1128Indicadores_TIC_2016_INTEF.pdf(Última consulta, enero 2016).

Lelouche, R. (1998). How education can benefit from computer: A critical review. Proceedings of IV International Conference CALISCE '98. Conferencia llevada a cabo en el congreso CALISCE'98, Donostia.

Liberati, A., Altman, D. G., Tetzlaff, J., Mulrow, C., Gøtzsche, P. C., Ioannidis J. P. A., Clarke, M., Devereaux, P. J., Kleijnen, J. \& Moher, D. (2009). The PRISMA statement for reporting systematic reviews and meta-analyses of studies that Evaluate health care interventions: Explanation and elaboration. Plos Medicine, 6 (7), e1000100. DOI: 10.1371. /journal.pmed.1000100

López, J. (2008). Innovar desde la base: Construir unidades didácticas globales en la sociedad de la información. Investigación en la Escuela, 66, 81-94. DOI: 10.12795/IE.2008.I66.06

López, J., Salvador, A. y De La Guardia, M. (1998): Estudio bibliométrico de la evolución de la revista 'Enseñanza de las Ciencias' a partir de sus fuentes de información. Enseñanza de las Ciencias, 16(3), 485-498.

Manchón-Gordón, A. y García-Carmona, A. (2018). ¿Qué investigación didáctica en el aula de física se publica en España? Una revisión crítica de la última década para el caso de educación secundaria. Enseñanza de las Ciencias. Revista de investigación y experiencias didácticas, 36 (2), 125. DOI: $10.5565 / \mathrm{rev} / \mathrm{ensciencias.2451}$

Manso, J. y Ezquerra, Á. (2014). Proyectos de investigación a través de la creación de audiovisuales: propuesta de actuación con alumnos del Programa de Diversificación Curricular. Revista Eureka sobre Enseñanza y Divulgación de las Ciencias, 11 (1), 54-67. DOI: 10.25267/Rev_Eureka_ensen_divulg_cienc.2014.v11.i1.06

Manso, J., Ezquerra, Á., Burgos, M. y Mafokozi, J. (2019). Análisis del tratamiento de contenidos en la creación de audiovisuales educativos. Revista Eureka sobre Enseñanza y Divulgación de las Ciencias, 16 (1), 1601.DOI: 10.25267/Rev_Eureka_ensen_divulg_cienc.2019.v16.11.1601

Marcelo, C., Yot, C. y Perera, V. (2016). El conocimiento tecnológico y tecnopedagógico en la enseñanza de las ciencias en la universidad. Un estudio descriptivo. Enseñanza de las ciencias: revista de investigación y experiencias didácticas, 34 (2), 67-86. DOI: $10.5565 / \mathrm{rev} / \mathrm{ensciencias.1552}$

Marín, N. (2010). Propuesta para compartir una base de datos de información bibliográfica (Bib) en didáctica de las ciencias. Revista Eureka sobre Enseñanza y Divulgación de las Ciencias, 7 (3), 613-635. DOI: 10.25267/Rev_Eureka_ensen_divulg_cienc.2010.v7.i3.02

Marques, A. y Reis, P. (2017). Producción y difusión de vídeos digitales sobre contaminación ambiental. Estudio de caso: Activismo colectivo basado en la investigación. Revista Eureka 
sobre Enseñanza y Divulgación de las Ciencias, 14(1), 215-226. DOI: 10.25267/Rev_Eureka_ensen_divulg_cienc.2017.v14.i1.16

Marrero, J.J. y Expósito, H. (2006). Metodología y las nuevas tecnologías. Revista Comunicación y Pedagogía (Primeras noticias), 211, 49-52.

Marrero, J.J. y Fernández, J. (2011). Aulas virtuales y los modelos didácticos en las ciencias experimentales. Revista Comunicación y Pedagogía (Primeras noticias), 254, 13-21.

Marrero-Galván, J. J. (2015). Elaboración de trabajos de indagación escolar usando herramientas de las Tecnologías de la Información y Comunicación: una propuesta didáctica. Revista Investigación en la Escuela, 87, 35-48. DOI: 10.12795/ie.2015.i87.03

Martínez-Pérez, A., y Lezcano-Barbero, F. (2020). Percepción del Impacto de la Covid-19 en los Profesionales de la Educación Social que Trabajan con Menores. Revista Internacional De Educación Para La Justicia Social, 9(3), 223-243. DOI: 10.15366/riejs2020.9.3.012

Martínez, C., Ibarra, A., Pérez, J. y Figueres, C. (2016). El uso de SIG de software libre en una práctica de Biología y Geología de 4o de ESO: los ecosistemas. Didáctica de las Ciencias Experimentales y Sociales, 30, 103-116. DOI: 10.7203/dces.30.4584.

Martins, C. y Campos, P. (2019). Sequência didática eletrônica com testes adaptativos para o ensino de Ecologia do Ensino Fundamental numa plataforma de ensino. Revista Electrónica de Enseñanza de las Ciencias, 18(1), 1-18.

Moreira, M. (1994). Diez años de la revista "Enseñanza de las Ciencias": de una ilusión a una realidad. Enseñanza de las ciencias: revista de investigación y experiencias didácticas, 12 (2), 147-153.

Navas, N. (2011). Utilización de un sistema Blended Learning en el módulo de energías renovables. Revista Eureka sobre Enseñanza y Divulgación de las Ciencias, 8(2), 171-179. DOI: 10.25267/Rev_Eureka ensen divulg cienc.2011.v8.i2.03

Negre, F. y Marín, V. (2014). Colaboración entre universidad y escuela: una experiencia a partir de la asignatura "diseño de proyectos tic". Investigación en la Escuela, 83, 69-80. DOI: 10.12795/IE.2014.I83.06

OCDE (2010). Educational Research and Innovation Are the New Millennium Learners Making the Grade? Technology Use and Educational Performance in PISA 2006, OCDE Publicaciones. $\mathrm{http}: / /$ recursostic.educacion.es/blogs/europa/media/blogs/europa/informes/Are_the_new_mill enium_learners_making_the_grade.pdf(Última consulta, enero 2016).

Ojeda-Barceló, F., Gutiérrez-Pérez, J. y Perales-Palacios, F. J. (2011). Diseño, fundamentos y validación de un programa virtual colaborativo en educación ambiental. Enseñanza de las ciencias: revista de investigación y experiencias didácticas, 29 (1), 127-146. DOI: $10.5565 / \mathrm{rev} / \mathrm{ec} / \mathrm{v} 29 \mathrm{n} 1.458$

Ojeda-Barceló, F., Gutiérrez-Pérez, J. y Perales-Palacios, F. J. (2017). ¿Qué herramientas proporcionan las Tecnologías de la Información y la Comunicación a la educación ambiental? Revista Eureka Sobre Enseñanza Y Divulgación De Las Ciencias, 6 (3), 318-344. Recuperado de https://revistas.uca.es/index.php/eureka/article/view/3677

Oñorbe, A. (2014). El uso de las TIC en el aula. Alambique Didáctica de las Ciencias Experimentales, 76, 5-7.

Pagani, D. y Da Silva, R. (2009). Formação inicial de professores de biologia: a prática colaborativa e o uso pedagógico do computador. Revista Electrónica de Enseñanza de las Ciencias, 8(2), $422-439$.

Pereira, A., Ostermann, F. y Cavalcanti, C. (2009). O ensino de Física Quântica na perspectiva sociocultural: uma análise de um debate entre futuros professores mediado por um interferômetro virtual de Mach-Zehnder. Revista Electrónica de Enseñanza de las Ciencias, 8(2), 376-398.

Pontes, A. (1999). Utilización del ordenador en la enseñanza de las ciencias. Alambique. Didáctica de las Ciencias Experimentales, 19, 53-64. 
Pontes, A. (2005a). Aplicaciones de las tecnologías de la información y de la comunicación en la educación científica. Primera parte: funciones y recursos. Revista Eureka sobre Enseñanza y Divulgación de las Ciencias, 2(1), 2-18.

Pontes, A. (2005b). Aplicaciones de las tecnologías de la información y de la comunicación en la educación científica. Segunda parte: aspectos metodológicos. Revista Eureka sobre Enseñanza y Divulgación de las Ciencias, 2(3), 330-343.

Prieto, R y Blanco, A. (1997). Las concepciones de los alumnos y la investigación en Didáctica de las Ciencias. Serv. Publ. Universidad de Málaga, Málaga.

Ramírez-Montoya, M.S. \& García-Peñalvo, F. (2018). Co-creation and open innovation: Systematic literature review. [Co-creación e innovación abierta: Revisión sistemática de literatura]. Comunicar, 54, 9-18. DOI: 10.3916/C54-2018-01

Redecker, C. y Punie, Y. (2017). European framework for the digital competence of educators DigCompEdu. Oficina de Publicaciones de la Unión Europea. DOI: 10.2760 / 159770

Romero, M. y Quesada, A. (2014). Nuevas tecnologías y aprendizaje significativo de las ciencias. Enseñanza de las ciencias: revista de investigación y experiencias didácticas, 32(1), 101-115.

Ruíz, A., Muñoz, A., Valladolid, M., Peña, J., Ruiz, A., Muñoz, A. y Peña, J. (2019). El iPad en la Educación científica de estudiantes de Secundaria y Bachillerato. Didáctica de las Ciencias Experimentales y Sociales, 36, 97-114. DOI: 10.7203/dces.36.12661

Sandia, B., Pérez, J. y Rivas, D. (2019). Propuesta metodológica para la creación de Objetos de Aprendizaje. Revista Electrónica de Enseñanza de las Ciencias, 18(3), 521-542.

Sanmartí, N. e Izquierdo, M. (2001). Cambio y conservación en la enseñanza de las ciencias ante las TIC. Alambique. Didáctica de las Ciencias Experimentales, 29, 71-83.

Santos, R. y Miranda, D. (2009). Formação de professores de Ciências e Física na Internet: porque um site de recomendação de conteúdos. Revista Electrónica de Enseñanza de las Ciencias, 8(1), 171-192.

Sola-Martínez, T., Cáceres-Reche, M. P., Romero-Rodríguez, J. M., y Ramos-Navas-Parejo, M. R. (2020). Estudio Bibliométrico de los documentos indexados en Scopus sobre la Formación del Profesorado en TIC que se relacionan con la Calidad Educativa. Revista Electrónica Interuniversitaria de Formación del Profesorado, 23(2). DOI: 10.6018/REIFOP.418611

Solbes, J., Fernández-Sánchez, J., Domínguez-Sales, M.C., Cantó, J. y Guisasola, J. (2018). Influencia de la formación y la investigación didáctica del profesorado de ciencias sobre su práctica docente. Enseñanza de las ciencias: revista de investigación y experiencias didácticas, 36(1), 25-44. DOI: $10.5565 / \mathrm{rev} / \mathrm{ensciencias.2355}$

Solbes, J., Souto, X.M. y Traver, M.J. (2004). El impacto de las nuevas tecnologías de la información y de la comunicación en el sistema escolar, Scripta Nova, 6(170), 71.

Solbes, J., Souto, X., Traver, M., Jardón, P. y Ramírez, S. (2004). Visión del alumnado de las TIC y sus implicaciones sociales, Investigación en la escuela, 54, 81-93.

Tobin, K. (1999). Internet como instrumento de formación de los maestros de ciencias: ¿agente transformador o catalizador de la reproducción cultural? Enseñanza de las ciencias: revista de investigación y experiencias didácticas, 17(2), 155-164.

Toma, R. B. (2020). Revisión sistemática de instrumentos de actitudes hacia la ciencia (2004- 2016). Enseñanza de las Ciencias, 38 (3), 143-159.160 DOI: 10-5565/rev/ensciencias.2854

Urrutia, G. y Bonfill, X. (2010). Declaración PRISMA: una propuesta para mejorar la publicación de revisiones sistemáticas y metaanálisis. Medicina clínica, 135(11), 507-511.

Valverde-Crespo, D. y González-Sánchez, J. (2016). Búsqueda y selección de información en recursos digitales: Percepciones de alumnos de Física y Química de Educación Secundaria Obligatoria y Bachillerato sobre Wikipedia. Revista Eureka sobre Enseñanza y Divulgación de las Ciencias, 13(1), 67-83. DOI: 10.25267/Rev_Eureka_ensen_divulg_cienc.2016.v13.i1.06

Villalustre, L., Del Moral, M. y Neira-Piñeiro, M. (2019). Percepción docente sobre la Realidad Aumentada en la enseñanza de ciencias en Primaria. Análisis DAFO. Revista Eureka sobre 
enseñanza $y$ divulgación de las ciencias, 16(3), 3301. DOI: 10.25267/rev_eureka_ensen_divulg_cienc.2019.v16.i3.3301

Cómo CITAR ESTE ARTÍ́CULO

Marrero Galván, J.J., Negrín Medina, M.A. y González Pérez, P. (2021). Las TIC en la didáctica de las ciencias en el ámbito español: revisión sistemática en relación con el tratamiento de competencias digitales. Didáctica de las ciencias experimentales y sociales, 41, 119-136. DOI: 10.7203/DCES.41.20260 